


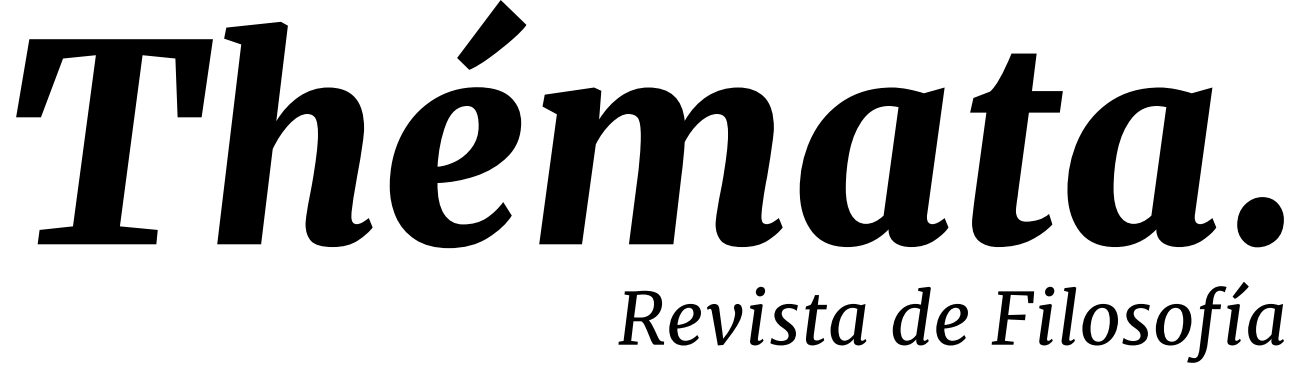

\section{La transmisión filosófica. Pensamiento de Javier Hernández-Pacheco Sanz}

Alejandro Martín Navarro (Coord.)

ESPECIAL THÉMATA

2022

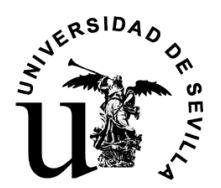

ISSN 0212-8365

e-ISSN 2253-900X

DOI: $10.12795 /$ themata

revistascientificas.us.es/index.php/themata

https://editorial.us.es/es/revistas/themata-revista-de-filosofia 
Thémata. Revista de Filosofía.

Número Extra

Thémata. Revista de Filosofía nace en el año 1983 con la intención de proporcionar a quienes investigan y producen en filosofía un cauce para publicar sus trabajos y fomentar un diálogo abierto sin condicionamientos ideológicos. En sus inicios participaron en el proyecto las Universidades de Murcia, Málaga y Sevilla, pero pronto quedaron como gestores de la revista un grupo de docentes de la Facultad de Filosofía de la Universidad de Sevilla.

Una preocupación constante de sus realizadores ha sido fomentar los planteamientos interdisciplinares. La revista ha estado abierta siempre a colaboradores de todas las latitudes y ha cubierto toda la gama del espectro filosófico, de lo que constituye una buena prueba la extensa nómina de autores que han publicado en sus páginas. En sus páginas pueden encontrarse trabajos de todas las disciplinas filosóficas: Historia de la Filosofía, Metafísica, Gnoseología, Epistemología, Lógica, Ética, Estética, Filosofía Política, Filosofía del Lenguaje, Filosofía de la Mente, Filosofía de la Ciencia, Filosofía de la Historia, Filosofía de la Cultura, etc. También ha querido ser muy flexible a la hora de acoger nuevos proyectos, fomentar discusiones sobre temas controvertidos y abrirse a nuevos valores filosóficos. Por esta razón, los investigadores jóvenes siempre han encontrado bien abiertas las puertas de la revista.

\section{Equipo editorial / Editorial Team Bases de Datos y Repertorios}

Director honorario

Jacinto Choza Armenta

jchoza@us.es

Director

Fernando Infante del Rosal

finfante@us.es

Director Adjunto

José Manuel Sánchez López

themata@us.es

Subdirectores

Jesús Navarro Reyes

jnr@us.es

Inmaculada Murcia Serrano

imurcia@us.es

Jesús de Garay

jgaray@us.es

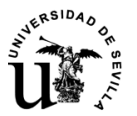

Facultad de Filosofía. Universidad de Sevilla

Departamento de Estética e Historia de la

Filosofía

Departamento de Filosofía y Lógica y Filosofía

de la Ciencia

Departamento de Metafísica y Corrientes

Actuales de la Filosofía, Ética y Filosofía Política

Camilo José Cela s/n, 41018 Sevilla (España)

e-mail: themata@us.es
Bibliográficas internacionales

Emerging Sources Citation Index (Web of

Science Group-Clarivate Analytics)

Dialnet (España)

Francis, Philosophie. INIST-CNRS (France)

Philosopher's Index (Bowling Green, OH, USA)

Directory of Open Access Journals (DOAJ)

Repertoire Bibliographique de Philosophie

(Louvain, Belgique)

Ulrich's Internacional Periodicals Directory

(New York, USA)

DialogJournalNameFinder (Palo Alto, CA, USA)

Periodicals Index Online (Michigan, USA)

Index Copernicus World of Journals

Gale-Cengage Learning-Informe Académico

Academic Journal Database

DULCINEA

Google Scholar

Electra

Bulletin signaletique. Philosophie, CNRS

(France)

Bibliográficas nacionales

ISOC - Filosofía. CINDOC (España)

De evaluación de la calidad de revistas

CARHUS Plus

ERIH PLUS Philosophy (2016)

REDIB

Latindex 2.0

MIAR

CIRC

DICE

Política editorial y directrices para autores/as, al final de la revista.

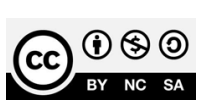


Thémata. Revista de Filosofía.

\section{Consejo Editor / Editorial Board}

\section{ARGENTINA}

Flavia Dezzuto, Universidad Nacional de Córdoba

ALEMANIA

Alberto Ciria, Munich

\section{CANADÁ}

Óscar Moro, University of New Found Land

CHILE

Mariano De la Maza, Universidad Católica de Chile

José Santos Herceg, Universidad de Santiago de Chile

\section{COLOMBIA}

Martha Cecilia Betancur García, Universidad de Caldas

Víctor Hugo Gómez Yepes, Universidad Pontificia Bolivariana

Gustavo Adolfo Muñoz Marín, Universidad Pontificia Bolivariana

\section{ESPAÑA}

Alfonso García Marqués, Universidad de Murcia

Antonio De Diego González, Universidad de Sevilla

Avelina Cecilia Lafuente, Universidad de Sevilla

Carlos Ortiz Landázuri, Universidad de Navarra

Celso Sánchez Capdequí, Universidad Pública de Navarra

Elena Ronzón Fernández, Universidad de Oviedo

Enrique Anrubia, Universidad CEU Cardenal Herrera

Federico Basáñez, Universidad de Sevilla

Fernando Wulff, Universidad de Málaga

Fernando M. Pérez Herranz, Universidad de Alicante

Fernando Pérez-Borbujo, Universitat Pompeu Fabra

Francisco Rodríguez Valls, Universidad de Sevilla

Ildefonso Murillo, Universidad Pontificia de Salamanca

Irene Comins Mingol, Universitat Jaume I Jacinto Rivera de Rosales Chacón, † UNED Joan B. Llinares, Universitat de València Jorge Ayala, Universidad de Zaragoza José Manuel Chillón Lorenzo, Universidad de Valladolid
Juan García González, Universidad de Málaga

Juan José Padial Benticuaga, Universidad de Málaga

Luis Miguel Arroyo Arrayás, Universidad de Huelva

Ma Luz Pintos Peñaranda, Universidad de Santiago de Compostela

Marcelo López Cambronero, Instituto de Filosofía Edith Stein

María del Carmen Paredes, Universidad de Salamanca

Octavi Piulats Riu, Universitat de Barcelona

Óscar Barroso Fernández, Universidad de Granada

Pedro Jesús Teruel, Universitat de València

Ramón Román Alcalá, Universidad de Córdoba

Ricardo Parellada, Universidad Complutense de Madrid

Sonia París Albert, Universitat Jaume I

Tomás Domingo Moratalla, UNED

\section{ESTADOS UNIDOS}

Witold Wolny, University of Virginia)

Thao Theresa Phuong Phan, University of Maryland

\section{REINO UNIDO}

Beatriz Caballero Rodríguez, University of Strathclyde

ITALIA

Luigi Bonanate, Università di Torino

\section{MÉXICO}

Rafael De Gasperín, Instituto Tecnológico de Monterrey

Julio Quesada, Universidad Veracruzana

Adriana Rodríguez Barraza, Universidad Veracruzana

PERÚ

Ananí Gutiérrez Aguilar, Universidad Nacional de San Agustín de Arequipa y Universidad Católica de Santa María

Nicanor Wong Ortiz, Universidad San Ignacio de Loyola

\section{PORTUGAL}

Yolanda Espiña, Universidade Católica Portuguesa

\section{TURQUÍA}

Mehmet Özkan, SETA Foundation for Political, Economic and Social Research 
Thémata. Revista de Filosofía.

\section{Comité Científico Asesor / Advisory Board}

ARGENTINA

Graciela Maturo, Universidad de Buenos Aires - CONICET

Jaime Peire, Universidad Nacional de Tres de Febrero- CONICET

\section{ALEMANIA}

Tomás Gil, Freie Universität Berlin

Fernando Inciarte, $\dagger$ Westfälische WilhelmsUniversität

Otto Saame, † Universität Mainz

\section{BULGARIA}

Lazar Koprinarov, South-West University 'Neofit Rilski'

CHILE

Carla Corduá, Universidad de Chile

Roberto Torreti, Universidad de Chile

COLOMBIA

Carlos Másmela, Universidad de Antioquía Fernando Zalamea, Universidad Nacional de Colombia

\section{ESPAÑA}

Agustín González Gallego, Universitat de Barcelona

Alejandro Llano, Universidad de Navarra Andrés Ortiz-Osés, Universidad de Deusto Ángel D'ors, † Universidad Complutense de Madrid

Antonio Hermosa Andújar, Universidad de Sevilla

Carlos Beorlegui Rodríguez, Universidad de Deusto

Concha Roldán Panadero, Instituto de Filosofía, CCHS-CSIC

Daniel Innerarity Grau, Ikerbasque, Basque Foundation for Science

Francisco Soler, Universidad de Sevilla Ignacio Falgueras, Universidad de Málaga Javier San Martín, UNED

Jesús Arellano Catalán, † Universidad de Sevilla

Joaquín Lomba Fuentes, Universidad de Zaragoza

Jorge Vicente Arregui, † Universidad de Málaga José María Prieto Soler, † Universidad de Sevilla José Rubio, Universidad de Málaga Juan Antonio Estrada Díaz, Universidad de Granada

Juan Arana Cañedo-Argüelles, Universidad de Sevilla
Luis Girón, Universidad Complutense de Madrid

Manuel Fontán Del Junco, Fundación March

Manuel Jiménez Redondo, Universitat de València

Marcelino Rodríguez Donís, Universidad de Sevilla

Miguel García-Baró López, Universidad Pontificia Comillas

Modesto Berciano, Universidad de Oviedo

Pascual Martínez-Freire, Universidad de Málaga

Rafael Alvira, Universidad de Navarra

Teresa Bejarano Fernández, Universidad de Sevilla

Vicente San Félix Vidarte, Universitat de València

\section{ESTADOS UNIDOS}

Lawrence Cahoone, University of Boston

FRANCIA

Nicolás Grimaldi, Université Paris IV-Sorbonne

\section{PARAGUAY}

Mario Ramos Reyes, Universidad Católica de Asunción

\section{REINO UNIDO}

Alexander Broadie, University of Glasgow

ISRAEL

Marcelo Dascal, † Tel Aviv University

ITALIA

Massimo Campanini, Università di Napoli l'Orientale

Maurizio Pagano, Università degli Studi del Piamonte Orientale. Amedeo Avogadro

JAPóN

Juan Masiá, Sophia University, Tokio

\section{MÉXICO}

Jaime Méndez Jiménez, Universidad Veracruzana

Ana Laura Santamaría, Instituto Tecnológico de Monterrey

Héctor Zagal, Universidad Panamericana

VENEZUELA

Seny Hernández Ledezma, Universidad Central de Venezuela

Thémata. Revista de Filosofía agradece la labor realizada desde su inicio por sus anteriores directores: Jacinto Choza, Juan Arana y Jesús de Garay. 


\section{Índice.}

\section{LA TRANSMISIÓN FILOSÓFICA. PENSAMIENTO DE JAVIER HERNÁNDEZ-}

PACHECO SANZ_ESPECIAL THÉMATA

9 El acto filosófico. En recuerdo de Javier Hernández-Pacheco Sanz Fernando Infante del Rosal

14 Presentación

Alejandro Martín Navarro (Coord.)

SEMBLANZAS Y RECUERDOS_JAVIER HERNÁNDEZ-PACHECO SANZ,

TRANSMISIÓN Y ENSEÑANZA DE LA FILOSOFÍA

19 Treinta y siete años con Pacheco. Variaciones sobre el cristianismo Jacinto Choza Armenta

$51 \quad$ Historia de un hombre sabio y bueno Juan Arana Cañedo-Argüelles

$54 \quad$ Una fuerza de la naturaleza José Manuel Sánchez López

58 In Memoriam a Javier Hernández-Pacheco Urbano Ferrer Santos

65 Recordando al profesor Javier Hernández-Pacheco Esperanza María Domínguez Sabido

OBRA Y PENSAMIENTO DE JAVIER HERNÁNDEZ-PACHECO SANZ_ARTÍCULOS

$71 \quad$ Una conversación pendiente Jesús de Garay Suárez-Llanos

87 Un viaje de ida y vuelta: de cómo el refugio se torna apertura. El proyectar(se) como apertura a lo Otro Elisa Fernández Bascones

107 Max Horkheimer y el anhelo de lo completamente Otro. (A propósito de la interpretación de la filosofía de Max Horkheimer de Javier Hernández-Pacheco) José Manuel Panea Márquez 
Thémata. Revista de Filosofía.

124 Javier Hernández-Pacheco: fe y filosofía

Lidia Romero Sánchez

$141 \quad$ La plenitud hacia la que tendemos: historia de una discusión truncada.

Francisco José Soler Gil

161 Tábula gratulatoria.

163 Política editorial.

166 Directrices para autores/as.

ILUSTRACIÓN DE CUBIERTA: Javier Infante 
Thémata. Revista de Filosofía.

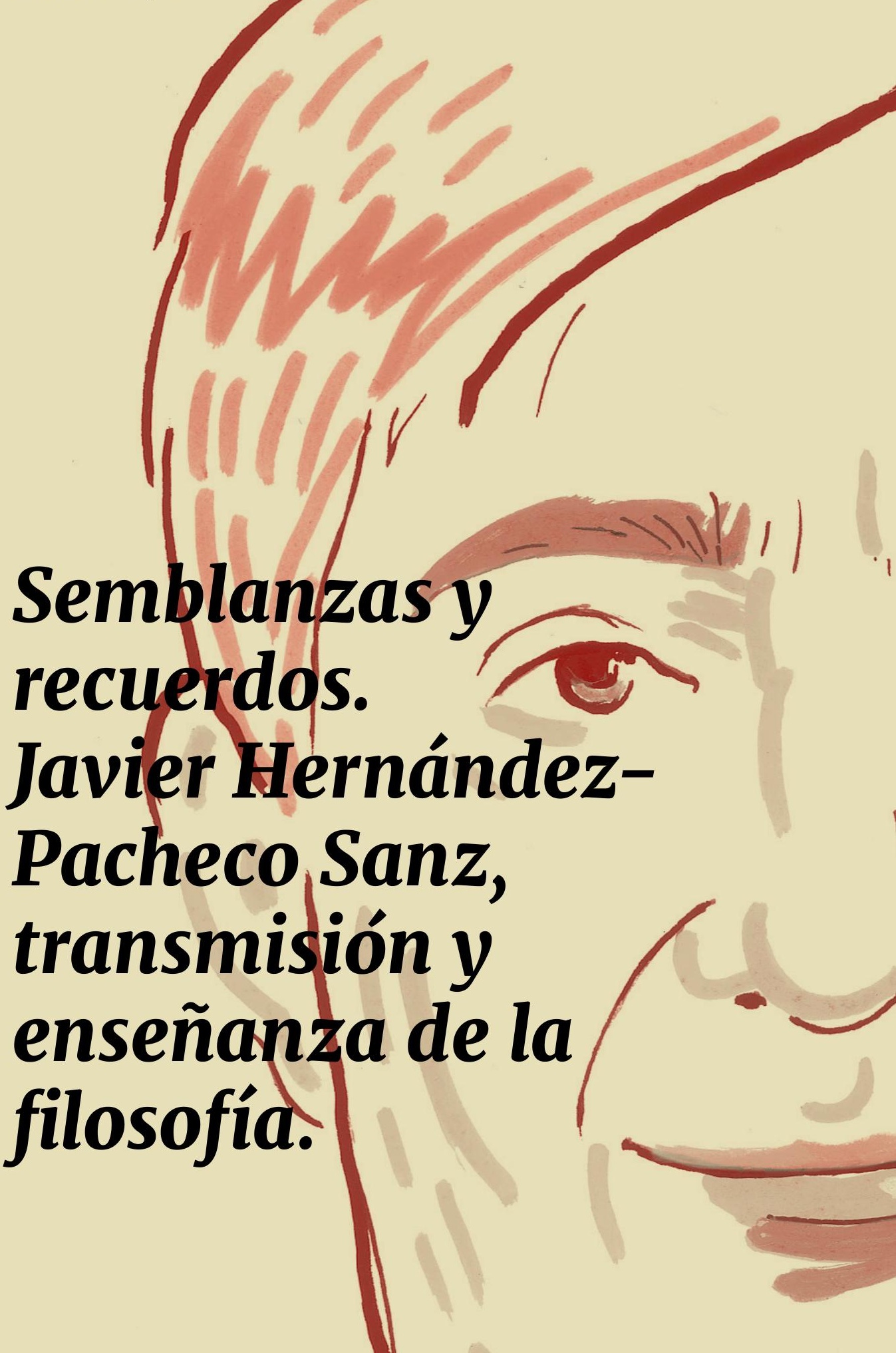




\title{
Historia de un hombre sabio y bueno.
}

\author{
Juan Arana Cañedo-Argüelles ${ }^{1}$ \\ Universidad de Sevilla, España
}

Debió ser allá por el año 82 más o menos. Estábamos poniendo en marcha la facultad de filosofía de la Hispalense. Un buen día apareció por allá un mozo alto y sonriente. Traía bajo el brazo dos tesis doctorales: una defendida en la Universidad Complutense sobre Tomás de Aquino; otra sobre Heidegger presentada, ien alemán!, ante la de Viena. Se llamaba Javier Hernández-Pacheco. Su llegada revolucionó el departamento. Nos enseñó que no era tan difícil hacer filosofía de verdad y además resultaba enormemente divertido. Así comenzaron unos años que no olvidarán quienes los vivieron. Horas y horas charlando sin parar en el viejo edificio de Puerta Osario... noches febriles de insomnio preparando clases, poniendo a punto artículos, libros, conferencias... autobuses fletados para desembarcar todos juntos, profesores y alumnos, en cualquier sitio donde hubiera algo interesante que oír y nuestra voz fuera escuchada... iniciativas como fundar una revista científica (o dos, o incluso tres), abrir una colección editorial, crear una sociedad de filosofía andaluza, un instituto de filosofía de la economía, una cátedra de filosofía alemana... No formábamos una escuela, ni creíamos que fuese bueno hacerlo. Cada cual tenía su propio proyecto y se abstenía de imponerlo a los demás. Tampoco hubiera sido fácil conseguirlo: todos éramos duros de mollera. Y la más dura de todas era la de Javier. Dura y rebosante de ideas: pretendía nada 
menos que elaborar una síntesis - genial, como todo lo suyo - del pensamiento clásico y el moderno. Aristóteles y Hegel eran las dos luminarias de la empresa y se proponía fusionarlos, resolviendo de paso todos los problemas de este atribulado mundo. Los colegas no estábamos dispuestos a dejarnos convencer, así que sin parar reiniciábamos la discusión, sin tregua ni punto final, semana tras semana, mes tras mes...

La filosofía por supuesto no lo era todo. También estaba la vida: nos casamos unos con otras, tuvimos hijos, apadrinamos recíprocamente nuestras criaturas. La fiesta continuaba; aquello no parecía tener fin. Sin embargo, lo tuvo: veinte o treinta libros y diez o doce niños después las cosas empezaron a enredarse, como siempre ocurre. En los tiempos difíciles que llegaron descubrí la entereza de mi amigo. No es solo una frase decir que las causas perdidas son las únicas por las que merece la pena luchar. Javier tenía un olfato infalible para detectarlas. Junto a él encajamos toda una gloriosa serie de derrotas. Ni nuestro modelo de universidad prosperó, ni tampoco triunfó la idea de filosofía que promovíamos. Pero pude aprender de él en qué consiste la lealtad a toda prueba, el fair play, la dolorida alegría de haber peleado hasta el final una buena batalla. Recuerdo una ocasión concreta: en un bar los dos lamíamos nuestras heridas (las suyas eran peores que las mías). Entró un lotero muy atildado (¿qué habrá sido de él?) y le espetó a Javier: "¡Caballero!... La solución a todos sus problemas... i40 millones!” Él le miró como diciendo: "Si usted supiera cuáles son mis problemas..." Curiosamente, la escena se repitió dos años después: acababa de ser padre de mellizos y exclamó con aquellos ojos chispeantes que iluminaban su rostro: “¡Ahora sí! ¡Véndame ese billete, por favor!"

Y es que entre tanto había renunciado a la insensata pretensión de cambiar el mundo. Eso sí, sin arriar sus pabellones. Javier, que tenía un alma romántica, usó de la elegancia a la hora de la retirada. No es posible conseguirlo todo: formular un sistema de pensamiento y difundirlo. Así que optó por lo primero, se olvidó de giras o mecenas y se concentró en las clases (siempre fueron magníficas) y en su obra. Ocupa más de 16 volúmenes en la recopilación que se completó hace poco. Ojalá que la Universidad de Sevilla tenga el buen juicio de conservar ese tesoro y ponerlo a la disposición de todos.

Javier era filósofo y era cristiano. Las dos cosas iban de la mano dentro de él. Asimismo se consideraba liberal en un sentido muy concreto: estaba 
tan firmemente convencido de su ideario, que le resultaba odiosa la perspectiva de imponer a nadie una presunta verdad o justicia por medio de leyes y reglamentos. También era un sevillano apasionado (de la provincia de Badajoz, como buena parte de nuestros conciudadanos). Al principio le costó aclimatarse, después de varios años en Centroeuropa. Recuerdo que un día me dijo: "iEsta es una ciudad donde no tiene sentido limpiarse los zapatos!" Por entonces la mitad de las aceras estaban aún pavimentadas con albero. Pero hasta el albero acabó gustándole... y meterse dentro de las bullas de Semana Santa persiguiendo un paso tras otro... y afiliarse a la hermanad rociera de Triana, de cuya junta directiva formó parte... y llevarla a Ella, cuando algún almonteño lo colaba debajo del paso los lunes por la mañana...

Era Pacheco un tipo tan colosal, que le gustaba hacerse pequeño. Hasta motejó de "gripecilla" al covid que lo llevó a la tumba. En la misa que ayer le celebramos pusieron delante del altar una urna minúscula con sus cenizas. Era imposible que un mocetón así cupiera dentro de ella y me sentí embargado por la emoción.

Querido Javier, desde ese Cielo en el que estás y sobre el que con tanto acierto has escrito, espero que seguirás enseñándonos a sobrellevar con alegría las penas de esta vida, empezando por la de no volver a verte entrar por la puerta con tu pantalón de pana y la bufanda al cuello. 


\section{Thémata. \\ Revista de Filosofía}

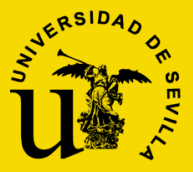

